\title{
Can Gravitational Waves Prevent Inflation?
}

\author{
HisA-AKI ShINKAI ${ }^{1}$ and KeI-ICHI MAEDA ${ }^{2}$ \\ Department of Physics, Waseda University, Shinjuku-ku, Tokyo 169-50, Japan
}

May 18th, 1993

\begin{abstract}
To investigate the cosmic no hair conjecture, we analyze numerically 1-dimensional plane symmetrical inhomogeneities due to gravitational waves in vacuum spacetimes with a positive cosmological constant. Assuming periodic gravitational pulse waves initially, we study the time evolution of those waves and the nature of their collisions. As measures of inhomogeneity on each hypersurface, we use the 3-dimensional Riemann invariant $\mathcal{I} \equiv{ }^{(3)} R_{i j k l}{ }^{(3)} R^{i j k l}$ and the electric and magnetic parts of the Weyl tensor. We find a temporal growth of the curvature in the waves' collision region, but the overall expansion of the universe later overcomes this effect. No singularity appears and the result is a "no hair" de Sitter spacetime. The waves we study have amplitudes between $0.020 \Lambda \leq \mathcal{I}^{1 / 2} \leq 125.0 \Lambda$ and widths between $0.080 l_{H} \leq l \leq 2.5 l_{H}$, where $l_{H}=(\Lambda / 3)^{-1 / 2}$, the horizon scale of de Sitter spacetime. This supports the cosmic no hair conjecture.
\end{abstract}

1 electronic mail: 62L508@jpnwas00.bitnet

2 electronic mail: maeda@jpnwas00.bitnet or maeda@cfi.waseda.ac.jp 
The widely accepted inflationary universe model[1] is an attempt to solve many longstanding cosmological difficulties - such as the flatness, horizon, and monopole problems - in the standard big-bang model. The explanation of the present isotropy and homogeneity of the universe are one of those problems. The inflationary scenario may resolve this problem by introducing a rapid cosmological expansion. But the scenario is usually studied in an isotropic and homogeneous spacetime, so that to determine whether inflation really isotropizes and homogenizes the universe and to see whether inflation is generic, we have to study an inflationary model in anisotropic and inhomogeneous spacetimes as well.

To explain the generality of inflation, the so-called cosmic no hair conjecture has proposed [2]. The conjecture is: "All initially expanding universes with positive cosmological constant $\Lambda$ approach the de Sitter spacetime asymptotically". There exist, however, simple counter examples. For example, a closed Friedmann-Robertson-Walker spacetime with a positive $\Lambda$ recollapses if the initial energy density is large enough. On the other hand, the de Sitter spacetime is stable against perturbations and many other models also support this conjecture, so that we expect we can prove a suitably refined version of this conjecture.

For homogeneous but anisotropic spacetimes, Wald [3] showed that, except in the Bianchi IX case, initial expansion and positive $\Lambda$ force an approach to the de Sitter spacetime within one Hubble expansion time $\tau_{H}=\sqrt{\Lambda / 3}$. And for Bianchi IX, the additional initial condition that $\Lambda$ be larger than one third of the maximum value of 3 -dimensional Ricci scalar leads to the same conclusion [3, 4]. For inhomogeneous spacetimes, on the other hand, the only practical method we have at present is to solve the Einstein equations numerically. Assuming a spherically symmetric spacetime, Goldwirth and Piran [5] studied the behavior of inhomogeneous distributions of a scalar field. They found a sufficient criterion for inflation that the physical scale of inhomogeneity of the scalar field be larger than several times the horizon scale.

We may, however, wonder whether this result is valid for cases with other symmetries or for more generic spacetimes. Assuming a plane symmetric spacetime, Kurki-Suonio et. al. [6] studied new inflationary model and found that inflation occurs only when the potential is flat enough. The spacetime they studied is, however, not sufficiently inhomogeneous in order to 
investigate the "no hair conjecture". This is the first reason why we study a plane symmetric spacetime in this paper.

Besides the inflaton field, there exist other sources of inhomogeneity, one of which is gravitational waves. Although any linear gravitational waves in de Sitter space will decay exponentially and desappear soon[7], we do not know what will happen if strong gravitational waves exist. If the strong gravitational waves are localized, those may collapse into black holes. Even if those are not localized as a plane symmetric case, such a strong gravitational field may evolve into a naked singularity. In fact, Szekeres and Khan-Penrose[8] showed that a collision of two plane symmetric gravitational waves in Minkowski background spacetime will form a singularity. We may wonder what will happen with strong gravitational waves in de Sitter background. A singularity may be formed and prevent inflation. In this report, we study such an inhomogeneity due to gravitational waves. Because they cannot exist in a spherically symmetric spacetime, gravitational wave inhomogeneities may play a different role in the homogenization process from that of the inflaton field analyzed previously. This is the second reason why we study a plane symmetric spacetime here.

Recently, Nakao et al. examined time symmetrical initial data for Brill waves in an axial symmetrical spacetime with cosmological constant and found that waves with large gravitational mass does not always provide trapped surfaces citenakaogw. They also found that a dust sphere with large gravitational mass in a background de Sitter spacetime does not collapse to form a black hole spacetime 10, and that there exists an upper bound on the area of the apparent horizon of a black hole in an asymptotically de Sitter spacetime 11], so that we may conjecture that a large inhomogeneity does not necessarily prevent cosmological constant-driven inflation. From these results, it seems plausible that in an inflationary era, inhomogeneities will simply evolve into many small black holes in a background de Sitter universe; the only worrisome possibility is that a naked singularity might form. Disposing of this worrisome possibility may thus become the main problem in the study of the cosmic no hair conjecture. Our 1-dimensional case seems well suited to address this problem. This is the third reason since a singularity formed in a plane symmetric spacetime is always naked. 
For the above three reasons, we have carried out a numerical study of inhomogeneities due to plane-symmetric gravitational waves with a cosmological constant. Since we treat a plane symmetric spacetime and gravitational waves, our simulation may be thought of a complementary work to the spherically symmetric one discussed by Goldwirth and Piran. Since we have not studied an inflaton field in this paper, however, our result may not be applied to a realistic universe as it is. (See also our comment in the references).

To analyze the behavior of 1-dimensional inhomogeneities due to gravitational waves, we assume spacetime is plane symmetric and vacuum. We also assume that a positive cosmological constant $\Lambda$ exists. Under these assumptions, we examine whether such a spacetime leads to an inflationary era, and whether such initial inhomogeneities and anisotropies smooth out during inflation periods.

We use the ADM formalism to solve the Einstein equation

$$
R_{\mu \nu}-\frac{1}{2} g_{\mu \nu} R+\Lambda g_{\mu \nu}=0, \text { labeleinstein }
$$

with the metric

$$
d s^{2}=-\left(\alpha^{2}-\beta^{2} / \gamma_{x x}\right) d t^{2}+2 \beta d t d x+\gamma_{x x} d x^{2}+\gamma_{y y} d y^{2}+\gamma_{y z} d y d z+\gamma_{z z} d z^{2}
$$

where the lapse function $\alpha$, the shift vector $\beta_{i}=(\beta, 0,0)$, and the 3-metric $\gamma_{i j}$ depend only on the time $t$ and propagation direction coordinate $x$.

We use the Hubble expansion time $\tau_{H}=\sqrt{\Lambda / 3}$ as our time unit, which is a characteristic expansion time of the expanding Universe. And our unit of length is also normalized to the horizon length of the de Sitter universe $l_{H}=1 / \sqrt{\Lambda / 3}$.

Our simulation procedure follows Nakamura et al. [12]: (i) Determine initial values by solving two constraint equations. (ii) Evolve time slices by using the dynamical equations. (iii) Check the results of (i) and (ii) using the two constraint equations on every time slice.

To determine the initial data, we use the 5th order Runge-Kutta method (Fehlberg method), and to integrate dynamical equations, we use a finite difference method with 400 meshes. In our calculation, the maximum errors in the Hamiltonian and momentum constraint equations are $O\left(10^{-4}\right)$ and $O\left(10^{-6}\right)$ on the initial hypersurface, respectively, and these accuracies are maintained even after evolution. 
We use the York-O'Murchadha's conformal approach 13] to get initial values. Setting $\gamma_{i j}=$ $\phi^{4} \hat{\gamma}_{i j}$, the Hamiltonian constraint equation becomes

$$
{ }^{8}{ }^{(3)} \hat{\Delta} \phi={ }^{(3)} \hat{R} \phi-\hat{A}_{i j} \hat{A}^{i j} \phi^{-7}+2\left(\frac{1}{3} \hat{K}^{2}-\Lambda\right) \phi^{5}
$$

where ${ }^{(3)} \hat{\Delta}$ is the 3 -dimensional Laplacian of a conformal metric $\hat{\gamma}_{i j},{ }^{(3)} \hat{R}$ is the 3 -dimensional scalar curvature, hat $A_{i j}$ is the trace-free part of the extrinsic curvature $\hat{K}_{i j}$ and hat $K(=K)$ is a trace part of $\hat{K}_{i j}$. The quantities with a hat denote physical variables in the conformal frame. In this approach, $\hat{\gamma}_{i j}, \hat{K}$ and the transverse- traceless(TT) part of the extrinsic curvature $\hat{K}_{i j}^{T T}$ are left to our choice.

We assume constant mean curvature on the initial hypersurface,

$$
\hat{K}=-\sqrt{3 \Lambda}\left(1+\delta_{K}\right)=\text { const. } \quad \text { on } \operatorname{Sigma}(t=0),
$$

where $\delta_{K}$ is a constant. Here, $\delta_{K}$ is introduced to find the periodic gravitational waves. $\delta_{K}=0$ is inconsistent with the presence of gravitational waves in the initial data. The reason is, when we have pulse-like inhomogeneities under periodic boundary condition, there are two energy sources; one is a positive cosmological constant and the other is the energy of pulse waves. These energy give contributions to the expansion of the universe, i.e. the first term in the r.h.s. of (田) is due to the cosmological constant, while the second one $\left(\delta_{K}\right)$ is due to the pulse waves.

With this slicing, the momentum constraint equation becomes trivial and $\hat{A}_{i j}=\hat{K}_{i j}^{T T}$. If we also impose $\phi \rightarrow 1$ at the numerical boundary edges, the value of $\delta_{K}$ is fixed; it is about $O\left(10^{-2}\right)$ in the physical frame.

For coordinate conditions, we impose a geodesic slicing condition such as $\alpha=1$ and $\beta=0$. This slicing condition entails the risk that our numerical hypersurface may hit a singularity and stop there. If no singularity appears, however, then it may be the best coordinate condition for revealing whether de Sitter space emerges as a result of time evolution.

As for the initial data, we consider two complementary cases:

[case 1] The inhomogeneities reside in the 3-metric gâmma $a_{i j}$ and $\hat{K}_{i j}^{T T}=0$,

[case 2] The 3-metric is conformally flat and the inhomogeneities reside in $\hat{K}_{i j}^{T T}$. 
In this Brief Reports, we present mainly the results of simulations for the [case 1], and we examine a pulse-like wave propagating in the $x$-direction, and expressed by the metric in York's conformal frame as

$$
\operatorname{diag}\left(\hat{\gamma}_{i j}\right)=\left(1,1+a \mathrm{e}^{-\left(x / x_{0}\right)^{2}}, 1\right)
$$

where $a$ and $x_{0}$ are free parameters. Varying these parameters, which we interpret as the wave's amplitude and width, we study the effects of a family of gravitational pulse waves on the spacetime structure.

As a measure of inhomogeneity, we first use the Riemann invariant scalar $\mathcal{I} \equiv{ }^{(3)} R_{i j k l}{ }^{(3)} R^{i j k l}$, where ${ }^{(3)} R_{i j k l}$ is the Riemann tensor of the 3 -metric on $\Sigma$, and we introduce a dimensionless variable normalized by the cosmological constant,

$$
\mathcal{C}(t, x) \equiv \frac{\mathcal{I}^{1 / 2}}{\Lambda} \quad \text { on } \Sigma(t),
$$

and call it the "curvature" hereafter. We estimate the magnitude of the inhomogeneities in the 3 -space $\Sigma$ by the maximum value of this "curvature" on each slice, i.e. $\mathcal{C}_{\max }(t)=$ $\max \left\{\left.\mathcal{C}(t, x)\right|_{x \in \Sigma}\right\}$

As we mentioned a moment ago, a pulse-like wave has two characteristic physical dimensions, a width and an amplitude. We use $\mathcal{C}_{\max }(t)$ as an amplitude measure, and we define the width $l(t)$ by the proper distance between two points where the trace $\gamma$ of 3 -metric $\gamma_{i j}$ (the square of the 3-volume) decreases by half from its maximum value $\gamma_{\max }$. When $a$ and $x_{0}$ are given, the initial form of a gravitational wave is determined, and the width $l(0)$ and $\mathcal{C}_{\max }(0)$ may be calculated.

In Figure 1, we show a typical time evolution of this model for the case $a=-0.10, x_{0}=$ $0.20 ; l=0.33, \mathcal{C}_{\max }(0)=1.25$. We see that the wave given on the initial surface propagates both in the $+x$ and $-x$ directions, and the "curvature" seems to be superposed in the interacting region of the waves. The spacetime, however, finally succumbs to the overall expansion driven by the cosmological constant, and becomes virtually indistinguishable from de Sitter spacetime within one Hubble expansion time $\tau_{H}$.

We also use 4-dimensional variables as a measure to see the homogenization process. In the vacuum spacetime, if the Weyl tensor $C_{\mu \nu}$ rho vanish, the spacetime is homogenized and 
isotropized. We use the decomposition of the Weyl tensor: $E_{\rho \sigma}=C_{\rho \mu \sigma \nu} n^{\mu} n^{\nu}, B_{\rho \sigma}={ }^{*} C_{\rho \mu \sigma \nu} n^{\mu} n^{\nu}$, where ${ }^{*} C_{\mu \nu \rho \sigma} \equiv \frac{1}{2} C_{\rho \text { sigma }}^{\alpha \beta} \varepsilon_{\alpha \beta \mu \nu}$ and $n^{\mu}$ is a timelike vector orthogonal to the hypersurface $\Sigma$. In analogy to electromagnetism, the 3-dimensional variables $E_{\rho \sigma}$ and $B_{\rho \sigma}$ are called an electric and a magnetic parts of the Weyl tensor, and we can reconstruct the Weyl tensor completely from this pair of tensors. We introduce

$$
\mathcal{H}(t, x)=E_{\mu \nu} E^{\mu \nu}+B_{\mu \nu} B^{\mu \nu}
$$

as a sort of gravitational "super-energy" (in fact the purely timelike component of the BelRobinson tensor 14]). If $\mathcal{H}(t, x) \rightarrow 0$, then $C_{\mu \nu \rho \sigma} \rightarrow 0$ unless the hypersurfaces become null. We find that $\mathcal{H}(t, x)$ almost vanishes within one Hubble expansion time, and that this indicates that our spacetime does indeed approach a homogeneous one (i.e. de Sitter spacetime).

In our simulation, $l$ and $\mathcal{C}_{\max }(0)$ range between $0.080 l_{H} \leq l \leq 2.5 l_{H}$ and $0.020 \leq \mathcal{C}_{\max }(0)$ leq80.0. From the present results, we conclude that for any large Riemann invariant and/or small width inhomogeneity on the initial hypersurface, the nonlinearity of the gravity has little effect and the spacetime always evolves into a de Sitter spacetime.

Jensen and Stein-Schabes [15] proved an extension of Wald's theorem, showing that inflation invariably eliminates all inhomogeneities consistent with a non-positive scalar 3-curvature. Such inhomogeneities are hardly generic, however. In our models, we find that ${ }^{(3)} R$ is initially negative, but it eventually becomes positive along the propagations of the distortions and finally ${ }^{(3)} R \rightarrow 0$ when the spacetime is homogenized (see Figure 2). This convinces us that the behavior we have found is not the case envisioned by Jensen and Stein-Schabes.

Next, we simulated collisions of two gravitational pulse waves. The motivation for such a simulation is the existence of exact solutions for colliding plane waves in which scalar curvature singularities occur. Szekeres and Khan-Penrose [8] were the first to find such solutions. Szekeres treats the collision of two thick or sandwich plane waves, while Khan-Penrose treat two $\delta$ function-like shock waves. These solutions show the nonlinearity of the gravity. Thus we interest in such a phenomena in more general and realistic situations.

It may be interesting to see whether singularities also occur when gravitational waves collide in the presence of a cosmological constant. To investigate this possibility, we construct initial 
data representing two nearby pulse waves. Since we impose periodic boundary conditions, there is no difference between the present case and the case with a single pulse waves in one half of our numerical region. But in the present setting the collision does not occur on the boundary, so it may be better to see the interaction of two waves. In Figure 3, a typical example of the time evolution of the "curvature" is shown. We see that the waves start to propagate in opposite directions $(+x$ and $-x)$ and then collide with each other. When the waves collide, the "curvature" is just superposed as in Fig.1, but more clearly. After the wave-crossing, each wave propagates away. (This behavior is reminiscent of solitonic waves.) Consequently, the spacetime is again homogenized by the expansion of the universe within one Hubble expansion time $\operatorname{tau}_{H}$. For a wide range of initial widths and "curvatures" $\left(0.080 l_{H} \leq l \leq 0.10 l_{H}\right.$, $40.0 \leq \mathcal{C}_{\max }(0) \leq 125.0$ and the periodic distance $d$ is $\left.0.20 l_{H} \leq d \leq 0.50 l_{H}\right)$, all inhomogeneities decay below $1 \%$ of their initial "curvatures" within one Hubble expansion time.

That these spacetime become homogeneous even if we include colliding waves is consistent with the calculations by Centrella and Matzner[16]. They examined the collision of gravitational shock waves in an expanding Kasner background both analytically and numerically, and concluded that such a collision leads to no singularity. The expansion due to the cosmological constant in our simulation is exponential - much faster than Kasner (power-law) expansion. Thus, our result would be expected from their result, although here we investigated pulse wave collisions systematically for a wide range of initial parameters and extracted the generic behavior of gravitational waves in expanding universe.

We also computed the evolution of [case 2] initial data, $\hat{\gamma}_{i j}=\delta_{i j}$ and $\operatorname{diag}\left(\right.$ hat $\left.K_{i j}^{T T}\right)=$ $\left(0, a \mathrm{e}^{-\left(x / x_{0}\right)^{2}},-a \mathrm{e}^{-\left(x / x_{0}\right)^{2}}\right)$, and found that all inhomogeneities present in them also decay within one Hubble expansion time. We also tried similar calculations with a different shape for the initial pulse waves, i.e., assuming a $\left[1+a \cos ^{2}\left(x / x_{0}\right)\right]$-form for the $\hat{\gamma}_{y y}$ components, and found the same results for time evolution. We conclude from this that our result is generic: all initial inhomogeneities decay within one Hubble expansion time and disappear.

The present result suggests no additional condition of the kind needed to make cosmic no hair conjecture a theorem, but it does give a wide class of examples in which gravitational 
inhomogeneities succumb to cosmological constant- driven expansion. We could not find any characteristic scale length. All inhomogeneous spacetimes expand to de Sitter universe. In this sence, our results are different from those of Goldwirth and Piran [5], who simulated effects of spherically symmetric inhomogeneities due to a realistic inflaton field. We do not yet know whether our results reflect the generic behavior of inhomogeneities driven by gravitational waves, or whether they are largely due to our ansatz of plane symmetry. In order to answer these questions, further simulations including a inflaton field, or more gravitational degrees of freedom - for example, those present in cylindrical or axially symmetrical, or even more general spacetimes - -are required. Such simulations are underway [17].

We would like to acknowledge Dr. L.Gunnarsen for useful discussions and critical reading of our manuscript. We also wish to thank K.Nakao and T.Nakamura for helpful discussions. This work was supported partially by the Grant- in-Aid for Scientific Research Fund of the Ministry of Education, Science and Culture Nos. (04234211 and 04640312), by a Waseda University Grant for Special Research Projects and by The Sumitomo Foundation. This work was one of the specific studies at the Center for Informatics, Waseda University. 


\section{References}

[1] The original idea is in

A.H. Guth, Phys. Rev. D23, 347 (1981);

K. Sato, Mon. Not. Roy. Astron. Soc. 195, 467 (1981).

[2] Reviews are, e.g.,

K.Maeda, in the Proceedings of 5th Marcel Grossman Meetings, ed. by R.Ruffini, p.145 (World Scientific, 1989);

K.Sato, in the Proceedings of I.A.U. Symposium No.130, The Large Scale Structure of the Universe, ed.by J.Audouze et al., p.67 (IAU, 1988)

[3] R.M.Wald, Phys. Rev. D28, 2118 (1983)

[4] Y.Kitada and K.Maeda, Phys. Rev. D45, 1416 (1991); to be published in Class. Quantum Grav.

[5] D.S.Goldwirth and T.Piran, Phys. Rev. D40, 3263 (1989); Phys. Rev. Lett. 64, 2852 (1990); Phys. Rep. 214, 223 (1992)

D.S. Goldwirth, Phys. Lett. B243, 41 (1990); Phys. Rev. D43, 3204 (1991)

[6] H.Kurki-Suonio, J.Centrella, R.A.Matzner and J.R.Wilson, Phys. Rev. D35, 435, (1987)

[7] e.g., T.Piran, in The Early Universe, ed. by W.Unruh, (Reidel,1986);

K.Nakao, T.Nakamura, K.Oohara and K.Maeda, Phys. Rev. D43, 1788 (1991)

[8] P.Szekeres, Nature 228, 1183 (1970); J. Math. Phys. 13, 286 (1972);

V.A.Khan and R.Penrose, Nature 229, 185 (1971).

[9] K.Nakao, K.Maeda, T.Nakamura and K.Oohara, Phys. Rev. D47 (1993) 3194.

[10] K.Nakao, Gen. Rel. Grav. 24, 1069 (1992)

[11] K.Nakao, K.Yamamoto and K.Maeda, Phys. Rev. D47 (1993) 3203;

T.Shiromizu, K.Nakao, H.Kodama and K.Maeda, Phys. Rev. D47 (1993) R3099

[12] T.Nakamura, K.Maeda, S.Miyama and M.Sasaki, Prog. Theo. Phys. 63, 1229 (1980)

[13] N.O'Murchadha and J.W.York,Jr., Phys. Rev. D10, 428 (1974)

[14] V.D.Zakharov, Gravitational Waves in Einstein's Theory, (Halsted Press, Jerusalem, 1973).

[15] L.G.Jensen and J.A.Stein-Schabes, Phys. Rev. D35, 1146 (1987)

[16] J.Centrella and R.A.Matzner, Astrophys. J. 230, 311 (1979); J.Centrella, Astrophys. J. 241, 875 (1980); J.Centrella and R.A.Matzner, Phys. Rev. D25, 930 (1982)

[17] We have just finished our simulation in a plane symmetric spacetime with a realistic inflaton field for a chaotic inflation model (H.Shinkai and K.Maeda, in preparation). We assumed the similar setting of initial data to those of Goldwirth and Piran, i.e., we added the radiation field to the inhomogeneous inflaton field to obtain a homogeneous total energy density initially. We have, however, found the similar results to that in the present paper, i.e., the inhomogeneous spacetimes which we have studied always approach to de Sitter universe. No naked singularity is formed. Hence our simulation suggests that our result is just because of a plane symmetric inhomogeneities but not because of those due to gravitational waves. 


\section{Figure Captions}

Figure 1: A typical example of the time evolutions of propagating plane waves. The "curvature" $\mathcal{C}(t, x)$ (see eq. (ब) ) is shown for the initial pulse shape of $l=0.33 l_{H}$ and $\mathcal{C}_{\max }(t=0)=1.25\left(a=-0.10, x_{0}=0.20\right.$ in eq. (5) $)$. We see the initial pulse propagates both in the $+x$ and $-x$ directions, but is smoothed out by the expansion of the universe within one Hubble expansion time.

Figure 2: The evolution of ${ }^{(3)} R$ at $x=0$ (center) for the same initial data as in Fig.1. This shows also the maximum deviations from zero at each time. We see that ${ }^{(3)} R$ does not remain negative for all time, so that our simulations are not governed by the case proven by Jensen and Stein-Schabes.

Figure 3: The time evolution of the "curvature" $\mathcal{C}(t, x)$ resulting from waves that are located closely. Two waves are the same form, $l=0.10 l_{H}$ and $\mathcal{C}_{\max }(t=0)=51.0$, and the periodic distance is $0.30 l_{H}$. We see them collide and in the collision region, the "curvature" seems to be superposed, but finally the spacetime is homogenized by the expansion of the universe. 